\title{
TERAPIA INTENSIVA NEONATAL E PEDIÁTRICA NO RIO DE JANEIRO: DISTRIBUIÇÄO DE LEITOS E ANÁLISE DE EQÜIDADE
}

\author{
Arnaldo Prata Barbosa*, Antônio José ledo Alves da Cunha, Edimilson Ramos Migowski de Carvalho, \\ Andréa Ferreira Portella, Miriam Perez Figueiredo de Andrade, Maria Clara de Magalhães Barbosa \\ Trabalho realizado no Instituto de Puericultura e Pediatria Martagão Gesteira (IPPMG), da UFRJ-Rio de Janeiro, RJ
}

RESUMO - OBJETIVos. Identificar as UTIs pediátricas do Rio de Janeiro, número de leitos, distribuição geográfica, natureza pública ou privada, tipo de atendimento e de hospital, estudar a demanda da população e elaborar propostas de melhoria na eqüidade.

Métodos. Foram visitadas todas as UTIs do Estado, de julho de 97 a junho de 98 , levantado o número de leitos e tempo médio de permanência. Com essas informações e dados do IBGE, estabeleceuse a necessidade de leitos e a comparação entre oferta e demanda por regiōes, formulando-se propostas de melhoria na eqüidade.

Resultados. Foram identificadas 80 UTIs (excluídas seis), totalizando 1.080 leitos; $60 \%$ intensivos e $40 \%$ semi-intensivos; $57 \%$ públicos e $43 \%$ privados; $52 \%$ em UTIs neonatais exclusivas, $14 \%$ em pediátricas e $34 \%$ em mistas (65\% leitos neonatais), totalizando 79 I leitos neonatais (73\%). A maioria das UTIs (75\%) situava-se em hospitais gerais, $20 \%$ em maternidades ou hospitais materno-infantis e somente $5 \%$ eram centros universitários; a maioria na região metropolitana (89\%), com $93 \%$ dos leitos para $74 \%$ da população infantil, dos quais a maioria na cidade do Rio de Janeiro (76\%), com $73 \%$ dos leitos para $37 \%$ da população, enquanto que no interior existem apenas oito unidades (II\%) e 79 leitos (7\%) para $26 \%$ da população.

Conclusōes. Não há eqüidade na distribuição e no acesso aos leitos disponíveis, com carência no setor público e excesso no privado, grande concentração na região metropolitana e somente $5 \%$ das UTIs em hospitais universitários, propondo-se medidas de redistribuição e alocação de novos leitos em regiões mais carentes, associada à criação de uma central de vagas e sistema eficiente de transporte e referência.

Unitermos: Cuidados intensivos. Saúde pública. Eqüidade. Acesso aos serviços de saúde. Estatísticas \& dados numéricos. Qualidade dos cuidados de saúde.

\section{INTRODUÇÃo}

As Unidades de Tratamento Intensivo (UTIs) tornaram-se um símbolo da moderna medicina, com inegável impacto na redução da mortalidade ${ }^{1-4}$. No Brasil, muito pouco se conhece da infra-estrutura disponível e, principalmente, da qualidade da assistência prestada por essas unidades. Recentemente, a Associação de Medicina Intensiva Brasileira (AMIB) lançou o "I Censo Brasileiro de UTIs", um projeto que visa conhecer melhor esta estrutura ${ }^{5}$. No entanto, por tratar-se de estudo cuja a participação das unidades é opcional, pode não refletir inteiramente a realidade da assistência disponível. No Estado do Rio de Janeiro, ainda hoje não se tem um conhecimento estruturado de variáveis básicas, como número de leitos em atividade versus demanda de atendimento, distribuição e regionalização desses leitos e características da população

*Correspondência:

Rua Siqueira Campos, 93-sala 1004-Copacabana Rio de Janeiro-RJ-CEP 22.03 1-070

Tel:(21)2256-1894-Fax:(21) 2256-1894 atendida. Em 1990, o Comitê de Emergência e Tratamento Intensivo da Sociedade de Pediatria do Estado do Rio de Janeiro (SOPERJ) apresentou em seu encontro científico daquele ano um pequeno estudo da situação existente na época nas unidades públicas da cidade do Rio de Janeiro ${ }^{6}$, e, mais recentemente, o Conselho Regional de Medicina do Estado do Rio de Janeiro (CREMERJ), através de seu Grupo de Trabalho Materno-Infantil, realizou um estudo abrangendo apenas as unidades neonatais públicas e privadas com leitos contratados pelo SUS 7 , mas não há registro, pelo menos de nosso conhecimento, de um estudo mais amplo, envolvendo todas as unidades, públicas e privadas, neonatais e pediátricas, que avalie a qualidade da assistência, tanto em seus aspectos estruturais, como em relação aos seus processos de assistência e resultados.

Visando construir um painel que responda a esses questionamentos essenciais, idealizouse o presente estudo, que tem como objetivos: I) identificar as UTIs neonatais e pediátricas do Estado, com o número de leitos instalados e em atividade, distribuindo-os de acordo com a região geográfica, áreas de planejamento de saúde, natureza pública ou privada, atendimento neonatal, pediátrico ou misto e tipo de hospital onde estão instaladas, contrapondo-os à demanda da população atendida; 2) elaborar propostas de melhoria na eqüidade.

\section{Métodos}

Foram estudadas todas as UTIs neonatais e pediátricas existentes no Estado do Rio de Janeiro durante os meses de julho de 1997 a junho de 1998, identificadas através de consulta aos registros do Serviço de Vigilância Sanitária da Secretaria de Estado de Saúde do Rio de Janeiro (SES-RJ), do CREMERJ, da SOPERJ, da Sociedade de Terapia Intensiva do Estado do Rio de Janeiro (SOTIERJ) e da Associação de Hospitais da Cidade e do Estado do Rio de Janeiro. Foram excluídas as UTIs que encontravam-se desativadas ou temporariamente fora de funcionamento durante o período de estudo.

Após a identificação das unidades, todas foram visitadas por uma equipe de trabalho da 
Tabela I -UTIs neonatais e pediátricas no Estado do Rio de Janeiro, em funcionamento no período de julho/97 a junho/98, distribuídas de acordo com a mesorregião geográfica, população, nascidos vivos/ano (por residência da mãe), natureza pública ou privada, tipo de atendimento e número total de leitos ativos.

\begin{tabular}{|c|c|c|c|c|c|c|c|c|c|c|c|}
\hline \multirow{2}{*}{$\begin{array}{l}\text { Mesorregião } \\
\text { (Regional de } \\
\text { Saúde) }\end{array}$} & \multirow{2}{*}{$\begin{array}{c}N^{0} \text { de } \\
\text { crianças } \\
(0-14 \text { anos })\end{array}$} & \multirow{2}{*}{$\begin{array}{l}\mathrm{N}^{0} \text { nascidos } \\
\text { vivos/ano }\end{array}$} & \multicolumn{2}{|c|}{$\mathrm{N}^{0}$ Unidades (\%) } & \multicolumn{3}{|c|}{ Tipo de atendimento (\%) } & \multirow{2}{*}{$\begin{array}{l}\text { Leitos } \\
\text { inativos } \\
\text { Total }\end{array}$} & \multicolumn{3}{|c|}{ Leitos ativos } \\
\hline & & & SUS & Privadas & Neo & Ped $^{2}$ & Misto & & UTI & UI & Total \\
\hline Baía da llha Grande & 36.850 & 2.888 & - & - & - & - & - & - & - & $\overline{-}$ & - \\
\hline Baixadas Litorâneas & 139.620 & 6.298 & - & $I(100,0)$ & - & - & $\mathrm{I}(100,0)$ & 4 & 8 & 0 & 8 \\
\hline Centro-Sul & 64.282 & 3.413 & - & - & - & - & & - & - & - & - \\
\hline Metropolitana & 2.584 .159 & 169.298 & $32(48,5)$ & $34(51,5)$ & $30(45,4)$ & $11(16,7)$ & $25(37,9)$ & 51 & 592 & 409 & 1001 \\
\hline Noroeste & 76.894 & 4.806 & - & - & - & - & - & - & - & - & - \\
\hline Norte & 191.000 & 12.334 & $\mid(50,0)$ & $I(50,0)$ & - & - & $2(100,0)$ & 0 & 18 & 4 & 22 \\
\hline MédioParaíba & 203.755 & 16.227 & I $(33,3)$ & $2(66,7)$ & $2(66,7)$ & - & $\mid(33,7)$ & 8 & 19 & 12 & 31 \\
\hline Serrana & 189.045 & 15.367 & I $(50,0)$ & $\mid(50,0)$ & & - & $2(100,0)$ & 2 & II & 7 & 18 \\
\hline Total & 3.485 .605 & 230.631 & $35(47,3)$ & $39(52,7)$ & $32(43,2)$ & II $(14,9)$ & $31(41,9)$ & 65 & 648 & 432 & 1080 \\
\hline
\end{tabular}

UTTs-Pediátricas exclusivas, gerais(atendem recém-nascidos eventualmente)ou cirúrgicas

Universidade Federal do Rio de Janeiro (UFRJ) em parceria coma Coordenação de Fiscalização Sanitária daSES-RJ, caracterizando-as como visitas oficiais de inspeção daqueleórgão de governo, garantindo-se assimo ocesso a todas as unidades eaverificação in loco das variáveis pesquisadas. Foi solicitado ainda aos médicos responsáveis que informassemotempomédiodepermanênciaem suas unidades, assim como o perfil de internações por faixa de peso nas unidades neonatais.

A seguir, as UTIs foram distribuídas por meso e microrregióes geográficas do Estado do Rio de Janeiro e por Regionais de Saúde, de acordo com a definição da Fundação Nacional de Saúde (FUNASA) ${ }^{8}$ e da Fundação Instituto Brasileiro de Geografia e Estatística (IBGE), assim como por áreas de planejamento de saúdee regiões administrativas, no caso do município do Rio de Janeiro, de acordo com a definição daSecretaria Municipal deUrbanismo da Prefeitura da cidade do Rio de Janeiro ${ }^{10}$. O acesso e a adequação às necessidades da população foram então pesquisados confrontando-se os dados colhidos com a necessidade projetada de leitos, a natureza pública ou privada e a distribuição populacional por região geográfica, de acordo com os dados do Censo de 1996 (IBGE)" . A necessidade projetada de leitos intensivos foi calculada da seguinte forma:

a) para os leitos neonatais levou-se em consideração o número de nascimentos, o percentual de recém-nascidos admitidos em UTI-Neonatal e a média de permanência (MP) dos pacientes na unidade, de acordo com proposição do Ministério da Saúde ${ }^{12}$, aplicando-se a seguinte fórmula:
LeitosdeUTI-Neonatal $=$

Nascidos vivos no período $\%$ de admissões emUTI $\times$ MP $\mathbf{N}^{\circ}$ de dias do período

A necessidade de leitos intensivos foi estabelecida em I/3 e de leitos semi-intensivos (unidade intermediária) em 2/3.

b) para os leitos pediátricos levou-se em consideração o número de internações na população em geral, o percentual de internações pediátricas, o percentual de admissões em UTI-Pediátrica e a média de permanência (MP), aplicando-se a seguinte fórmula:

\section{Leitos de UTIP =}

Int. noperíodox\% int.pediátricas $\mathrm{\%}$ \%admissóesemUTI x MP $\mathbf{N}^{\circ}$ de dias do período

c) o percentual da população com acesso à medicina suplementar privada foi estabelecido em $25 \%$, a partir de informações sobreo número de beneficiários disponibilizadas pela Associação Brasileira de Medicina de Grupo $\left(\right.$ ABRAMGE) ${ }^{13}$ em relação ao total da população brasileirainformado pelo IBGE".

A partir dos dados levantados, procurouse elaborar propostas de melhoria na eqüidade do sistema.

O projeto foi aprovado pela Comissão de Ética em Pesquisa do Instituto de Puericultura e Pediatria Martagão Gesteira (IPPMG) da UFRJ. As UTIs e respectivas instituições estudadas não tiveram a identificação revelada no decorrer do trabalho e na apresentação dos resultados, sendo caracterizadas através de numeração aleatória.

\section{Resultados}

Foram identificadas 80 UTls neonatais e pediátricas em todo o Estado, sendo que seis foram excluídas por encontrarem-se desativadas ou temporariamente fora de funcionamento. Permaneceram 74 unidades. A distribuição regional, comparada com a população de crianças entre 0 e 14 anos, número de nascidos vivos por ano (por residência da mãe), natureza pública (pacientes do SUS) ou privada, tipo de atendimento e número total de leitos ativos encontra-se sintetizada na Tabela $\mathrm{I}$. O número de leitos ativos é de $\mathrm{I} .080$, compreendendo 648 leitos de UTI $(60,0 \%)$ e 432 leitos de $\mathrm{UI}(40,0 \%)$. A relação entre o número de leitos ativos, públicos e privados, e a distribuição populacional por região geográfica de saúde é apresentada na Tabela 2. 0 número de leitos inativos foi da ordem de 65 $(5,8 \%)$. No entanto, em duas unidades o número de leitos ativos superou em 19 o número de leitos programados, o que resultou em um total de I. 080 (95,9\%) leitos ativos (capacidade operacional) para I. 126 leitos planejados.

Adistribuição destes leitos entre públicos e privados foi da ordem de 616 (57,0\%) e 464 $(43,0 \%)$, respectivamente. Por outro lado, a distribuição destes leitos entre neonatais, pediátricos (gerais, exclusivos ou cirúrgicos) e mistos correspondeu a $557(5 \mathrm{I}, 6 \%), 150$ $(13,9 \%)$ e 373 (34,5\%), respectivamente, conforme pode ser observado de modo mais detalhado na Tabela 3.

A maior concentração de unidades foi observada na região metropolitana (Tabela 4) e a distribuição das 50 unidades localizadas 
Tabela 2 - Relação entre número de leitos ativos e população infantil por região geográfica de saúde

\begin{tabular}{|c|c|c|c|c|c|c|c|}
\hline \multirow[t]{2}{*}{ Mesorregião } & \multirow{2}{*}{$\begin{array}{l}\mathrm{N}^{0} \text { decrianças } \\
\text { (0 a I4 anos) }\end{array}$} & \multicolumn{3}{|c|}{$N^{0}$ de leitos ativos } & \multicolumn{3}{|c|}{ Relação Nº Leitos/População } \\
\hline & & Públicos & Privados & Total & Públicos & Privados & Total \\
\hline Baía da llha Grande & 36.850 & - & - & - & - & - & $0: 36.850$ \\
\hline Baixadas Litorâneas & 139.620 & - & 8 & 8 & $0: 105.000$ & | : 4.350 & $1: 17.452$ \\
\hline Centro-Sul & 64.282 & - & - & - & - & - & $0: 64.282$ \\
\hline Metropolitana & 2.584 .159 & 582 & 419 & 1.001 & $1: 3.330$ & $|:| .54 \mid$ & $|: 2.58|$ \\
\hline Noroeste & 76.894 & - & - & - & - & - & $0: 76.894$ \\
\hline Norte & 191.000 & 10 & 12 & 22 & $1: 14.300$ & I:4.000 & $1: 8.68 \mid$ \\
\hline MédioParába & 203.755 & 12 & 19 & 31 & $1: 12.700$ & $1: 2.700$ & $1: 6.752$ \\
\hline Serrana & 189.045 & 12 & 6 & 18 & $1: 11.800$ & | : 7.900 & $1: 10.502$ \\
\hline Total & 3.485 .605 & 616 & 464 & 1.080 & I: 4.250 & I : I.880 & I : 3.227 \\
\hline
\end{tabular}

\begin{tabular}{|c|c|c|c|c|c|c|c|c|c|}
\hline \multirow[t]{3}{*}{ Mesorregião } & \multirow{3}{*}{$\begin{array}{c}\text { População } \\
\text { de } 0-14 \text { anos }\end{array}$} & \multicolumn{2}{|c|}{ Leitos Neonatais } & \multicolumn{2}{|c|}{ Leitos Pediátricos } & \multicolumn{4}{|c|}{ Leitos mistos } \\
\hline & & \multirow[t]{2}{*}{$\underline{\text { SUS }}$} & \multirow[t]{2}{*}{ Privados } & \multirow[t]{2}{*}{ SUS } & \multirow[t]{2}{*}{ Privados } & \multicolumn{2}{|c|}{ SUS } & \multicolumn{2}{|c|}{ Privados } \\
\hline & & & & & & Neo & Ped & Neo & Ped \\
\hline Baía da llha Grande & 36.850 & - & - & - & - & - & - & - & - \\
\hline BaixadasLitorâneas & 139.620 & - & - & - & - & - & - & 5 & 3 \\
\hline Centro-Sul & 64.282 & - & - & - & - & - & - & - & - \\
\hline Metropolitana & 2.584 .159 & 343 & 196 & 146 & 4 & 60 & 33 & 134 & 85 \\
\hline Noroeste & 76.894 & - & - & - & - & - & - & - & - \\
\hline Norte & 191.000 & - & - & - & - & 2 & 8 & 10 & 2 \\
\hline Médio Paraíba & 203.755 & 12 & 6 & - & - & - & - & 9 & 4 \\
\hline Serrana & 189.045 & - & - & - & - & 10 & 2 & 4 & 2 \\
\hline Total & 3.485 .605 & 355 & 202 & 146 & 4 & 72 & 43 & 162 & 96 \\
\hline
\end{tabular}

Tabela 4 - Distribuição UTIs e leitos na região metropolitana, população infantil e número de nascidos vivos por ano (por residência materna)

\begin{tabular}{|c|c|c|c|c|c|c|c|}
\hline \multirow[t]{2}{*}{ Município } & \multirow{2}{*}{$\begin{array}{l}\text { Crianças de } \\
0-14 \text { anos }^{\mathrm{a}}\end{array}$} & \multirow{2}{*}{$\begin{array}{l}\text { Nascidos vivos/ano } \\
\text { (residência da mãe) }\end{array}$} & \multirow[b]{2}{*}{ UTIs } & \multicolumn{2}{|c|}{ Leitos do SUS } & \multicolumn{2}{|c|}{ Leitos Privados } \\
\hline & & & & $\mathrm{Neo}$ & Ped & Neo & Ped \\
\hline BelfortRoxo & 127.556 & 7.053 & - & - & - & - & - \\
\hline Duquede Caxias & 210.105 & 5.478 & 2 & - & - & 28 & 8 \\
\hline Guapimirim & 9.918 & 676 & - & - & - & - & - \\
\hline Itaborá & 54.540 & 3.619 & - & - & - & - & - \\
\hline Itaguaíc & 37.616 & 2.084 & - & - & - & - & - \\
\hline Japeri & 24.246 & $1.57 \mid$ & - & - & - & - & - \\
\hline Magé & 55.435 & 3.866 & - & - & - & - & - \\
\hline Mangaratiba & 5.773 & 432 & - & - & - & - & - \\
\hline Maricá & 16.113 & 740 & - & - & - & - & - \\
\hline Nilópolis & 39.272 & 2.838 & I & - & 46 & - & - \\
\hline Niterói & 98.417 & 7.732 & 6 & 29 & 5 & 36 & 8 \\
\hline Novalguaçu & 241.338 & 10.558 & 4 & 25 & 45 & II & - \\
\hline Paracambi & 10.178 & 752 & - & - & - & - & - \\
\hline Queimados & 33.954 & 2.899 & - & - & - & - & - \\
\hline Rio de Janeiro & 1.282 .308 & 95.894 & 50 & 337 & 78 & 246 & 73 \\
\hline SãoGonçalo & 216.237 & 18.324 & 3 & 12 & 5 & 9 & - \\
\hline S.João de Meriti & 121.153 & 4.782 & - & - & - & - & - \\
\hline TOTAL & 2.584 .159 & 169.298 & 66 & 403 & 179 & 330 & 89 \\
\hline
\end{tabular}

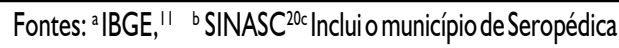


Tabela 5 - UTIs neonatais e pediátricas no município do Rio de Janeiro, em funcionamento no período de julho/97 a junho/98, distribuídas de acordo com a área de planejamento de saúde, população, natureza pública ou privada, tipo de atendimento e número total de leitos ativos

\begin{tabular}{|c|c|c|c|c|c|c|c|c|c|}
\hline \multirow[t]{2}{*}{ Área de Planejamento } & \multirow{2}{*}{$\begin{array}{c}N^{0} \text { de } \\
\text { crianças } \\
(0-14 \text { anos })\end{array}$} & \multicolumn{2}{|c|}{$\begin{array}{l}N^{0} \text { de Unidades } \\
\text { (n } n^{0} \text { leitos ativos) }\end{array}$} & \multicolumn{3}{|c|}{$\begin{array}{l}\text { Tipo de atendimento } \\
\text { ( } \mathrm{n}^{\circ} \text { de leitos ativos) }\end{array}$} & \multicolumn{3}{|c|}{$\mathbf{N}^{0}$ de leitos ativos } \\
\hline & & SUS & Privadas & Neonatal & Pediátrico $^{\mathrm{a}}$ & Misto & $\overline{\mathrm{UTI}}$ & UI & Total \\
\hline AP I (Centro/ Portuária) & 59.379 & $\overline{140}$ & 22 & 120 & II & 31 & $\overline{82}$ & 80 & 162 \\
\hline AP2.2(Zona Norte) & 66.545 & 36 & 60 & 34 & 18 & 44 & 62 & 34 & 96 \\
\hline AP3.I (Ramos, Penha,llha) & 194.366 & 16 & 27 & - & - & 43 & 36 & 7 & 43 \\
\hline AP4 (Jacarepaguá, Barra) & 133.154 & 23 & 39 & 47 & 8 & 7 & 36 & 26 & 62 \\
\hline AP5.I (Bangu) & 160.422 & - & 45 & - & - & 45 & 37 & 8 & 45 \\
\hline AP5.2(CampoGrande) & 114.138 & 24 & 9 & 20 & 4 & 9 & 22 & $\|$ & 33 \\
\hline AP5.3 (Santa Cruz/Guaratiba) & 104.883 & - & - & - & - & - & 0 & 0 & 0 \\
\hline Total & I.282.308 & 415 & 319 & 452 & 59 & 223 & 437 & 297 & 734 \\
\hline
\end{tabular}

aTIs-Pediátricas exclusivas, gerais(atendem recém-nascidos eventualmente)oucirúrgicas.

\begin{tabular}{|c|c|c|c|c|c|}
\hline $1.500 \mathrm{a} 2.499 \mathrm{~g}$ & 18.088 & $7,8 \%$ & $3.577(19,8 \%)$ & 1,95 & $40 \%$ \\
\hline$\geq 2.500 \mathrm{~g}$ & 206.990 & $89,7 \%$ & $3.158(1,5 \%)$ & 1,95 & $3 \%$ \\
\hline Ignorado & 2.749 & $1,2 \%$ & - & - & - \\
\hline
\end{tabular}

aFonte: SINASC, DATASUS para o ano de 1996, no estado do Rio de Janeiro.

bapenas das unidades que informaram seus indicadores.

no município do Rio de Janeiro de acordo com a área de planejamento de saúde é apresentada na Tabela 5.

Quanto ao tipo de hospital, observou-se que a maioria das unidades $(50)$ está localizada em hospitais gerais $(67,6 \%)$; oito $(10,8 \%)$ em maternidades; sete $(9,5 \%)$ em hospitais materno-infantis; cinco $(6,7 \%)$ em hospitais pediátricos gerais e quatro $(5,4 \%)$ em clínicas de emergência pediátrica. Observou-se ainda que somente quatro unidades $(5,4 \%)$ estão localizadas em hospitais universitários.

Em relação ao cálculo do número de leitos necessários, a aplicação da metodologia descrita considerou para os leitos neonatais o número de nascidos vivos de acordo com as estatísticas vitais fornecidas pelo Sistema de Informações de Nascidos Vivos (SINASC) ${ }^{14}$ para o ano de 1996 (total de 230.750 nascimentos, por residência da mãe) naquele ano. Para o percentual de admissões na UTI, considerou-se o percentual de 7\%(Tabela 6), onde o fator de correção utilizado foi calculado partindo-se da premissa de que 100\% dos recémnascidos com peso de nascimento igual ou inferior a $1.500 \mathrm{~g}$ deveriam ter sido admitidos em UTI-Neonatal. Para a média de permanência, considerou-se 20 dias, valor próximo à mediana do último quartil da variação encontrada neste trabalho (19 dias). A aplicação destes dados na fórmula proposta indicou a necessidade de 885 leitos de UTI-Neonatal no Estado, ou aproximadamente 900 leitos, dos quais 300 intensivos e 600 semi-intensivos.

Em relação à necessidade de leitos de UTI-Pediátrica, a aplicação da fórmula descrita tomou como base as seguintes premissas: I) 0 parâmetro de 0,I internações por habitante por ano, internacionalmente recomendado para cobertura assistencial à população em geral nos países em desenvolvimento ${ }^{15}$; 2 ) os dados obtidos através do DATASUS ${ }^{16}$ indicando que o número de internações pelo SUS no Brasil em I 996 foi de II.932.654 e os dados fornecidos pelaABRAMGE ${ }^{13}$ de que o número de internações na medicina suplementar privada foi de 4.110 .000 naquele mesmo ano, para uma população de 157.070.163 (IBGE)", o que ratificou o índice geral de 0 , I internações por habitante por ano; 3) a população no Estado do Rio de Janeiro, que no ano de 1996 foi de |3.406.308 (IBGE) ${ }^{\prime 1}$; 4) o percentual de internações pediátricas, que no SUS foi de I.558.702 para um total de II.932.654 $\left.(13 \%)^{16} ; 4\right)$ a recomendação do Ministério da Saúde de que o quantitativo de leitos intensivos deve ser de $6 \%$ dos leitos de internações pediátricas em geral ${ }^{17}$; 5) a média de permanência de oito dias, de acordo com a mediana dos serviços públicos (UTIs-Pediátricas) que informaram seus indicadores neste trabalho. Levando-se em consideração estes dados, chegou-se ao cálculo de 230 leitos necessários emUTI-Pediátrica.

Portanto, o número total de leitos intensivos (neonatais e pediátricos) previstos como 
Tabela 7 - Proposta de distribuição de novos leitos de terapia intensiva neonatal e pediátrica a serem criados ou remanejados no Estado do Rio de Janeiro, de acordo com a região geográfica

\begin{tabular}{|c|c|c|c|c|c|c|}
\hline Regional de Saúde & Cidade & Tipo de UTI & UTI Neo & UINeo & UTI Ped & Total \\
\hline Baía da llha Grande & Angrados Reis & UTI-mista & 3 & 4 & 3 & 10 \\
\hline \multirow[t]{3}{*}{ Baixadas Litorâneas } & RioBonito & UTI-neonatal & 2 & 3 & - & 5 \\
\hline & CaboFrio & UTI-neonatal & 4 & 6 & - & 10 \\
\hline & & UTI-pediátrica & - & - & 10 & 10 \\
\hline Centro-Sul & Três Rios $^{a}$ & UT1-mista & 3 & 4 & 3 & 10 \\
\hline \multirow[t]{2}{*}{ Noroeste } & Itaperuna & UTI-neonatal & 5 & 10 & - & 15 \\
\hline & & UTI-pediátrica & - & - & 10 & 10 \\
\hline \multirow[t]{2}{*}{ Norte } & Campos & UTI-neonatal & 8 & 12 & - & 20 \\
\hline & Macaé & UTI-mista & 4 & 4 & 2 & 10 \\
\hline \multirow[t]{3}{*}{ MédioParaíba } & VoltaRedonda & UTI-neonatal & 4 & 6 & - & 10 \\
\hline & & UTI-pediátrica & - & - & 10 & 10 \\
\hline & BarraMansa & UTI-neonatal & 4 & 6 & - & 10 \\
\hline \multirow{4}{*}{ Serrana } & Friburgo & UTI-neonatal & 4 & 6 & - & 10 \\
\hline & & UTI-pediátrica & - & - & 5 & 5 \\
\hline & Teresópolis & UTI-neonatal & 4 & 6 & - & 10 \\
\hline & & UTI-pediátrica & - & - & 5 & 5 \\
\hline \multirow[t]{8}{*}{ Metropolitana } & Itaguaí & UTI-mista & 3 & 4 & 3 & 10 \\
\hline & Duquede Caxias & UTI-neonatal & 8 & 12 & - & 20 \\
\hline & & UTI-neonatal & 4 & 6 & - & 10 \\
\hline & & UTI-neonatal & 4 & 6 & - & 10 \\
\hline & SJoãode Meriti & UTI-neonatal & 8 & 12 & - & 20 \\
\hline & Novalguaçu & UTI-neonatal & 8 & 12 & - & 20 \\
\hline & Nilópolis & UTI-neonatal & 2 & 4 & - & 6 \\
\hline & Niterói (HUAP) $)^{b}$ & UTI-pediátrica & - & - & 5 & 5 \\
\hline \multirow{2}{*}{ Rio de Janeiro } & UFRJ(IPPMG) & UT1-mista & 2 & 3 & 13 & 18 \\
\hline & UER) $(H U P E)^{b}$ & UTI-pediátrica & - & - & 5 & 5 \\
\hline \multirow[t]{2}{*}{ AP 5.3} & Hospital Pedroll & UTI-neonatal & 8 & 12 & - & 20 \\
\hline & & UTI-pediátrica & - & - & 6 & 6 \\
\hline AP 3.3 & HCarlos Chagas & UTI-pediátrica & - & - & 10 & 10 \\
\hline \multirow{3}{*}{ AP 5.1} & HAlbertSchweitzer & UTI-neonatal & 8 & 12 & - & 20 \\
\hline & & UTI-pediátrica & - & - & 10 & 10 \\
\hline & & & $100^{c}$ & $150^{c}$ & $100^{d}$ & 350 \\
\hline
\end{tabular}

${ }^{a}$ ou na cidade de Vassouras, se localizada em hospital universitário.

${ }^{b}$ recomendado por ser hospital universitário.

' $80 \%$ de leitos novos; $20 \%$ poderiam ser remanejados.

${ }^{\mathrm{S}} 50 \%$ de leitosnovos (interior); $50 \%$ poderiam ser remanejados (região metropolitana)

necessários no Estado do Rio de Janeiro, com base nas estatísticas vitais do ano de 1996 e nos resultados deste trabalho, pode ser considerado como de aproximadamente I.I30, o que para uma população de 3.485 .605 crianças de 0 a 14 anos de idade representa uma relação geral aproximada de um leito para cada 3.000 crianças. A distribuição destes leitos deve corresponder a aproximadamente $80 \%$ de leitos neonatais (aproximadamente 1:4.000 crianças) e $20 \%$ de leitos pediátricos (aproximadamente I:I5.000 crianças), sendo $75 \%$ de leitos públicos e $25 \%$ de leitos privados e aproximadamente $35 \%$ de leitos intensivos e $65 \%$ de leitos semi-intensivos.
Como propostas para melhoria na eqüidade do sistema, sugerem-se as seguintes medidas: I) criação de um sistema de referência e controle centralizado das vagas disponíveis; 2 ) sistema de transporte efetivo; 3 ) remanejamento de leitos existentes e criação de novos leitos, conforme apresentado na Tabela 7.

\section{Discussão}

Em relação à necessidade de leitos neonatais, costuma-se considerar que seriam necessários em média um leito intensivo e três semi-intensivos para cada 1.000 nascimentos por ano ${ }^{18}$. Este critério, de caráter bastante prático, na verdade depende da freqüência de nascimentos prematuros e de alto risco com indicação de UTI, havendo grandes diferenças entre os diversos países, entre regiões de um mesmo país e até entre instituições de uma mesma região. Enquanto nos países industrializados, por exemplo, a taxa de recém-nascidos de baixo peso $(<2.500 \mathrm{~g})$ é em média de $6 \%$, nos países em desenvolvimento é de $19 \%$ e nos países menos desenvolvidos de $24 \%{ }^{19}$ De acordo com o Centro Latino-Americano de Perinatologia e Desenvolvimento (CLAP), este percentual na América Latina e Caribe é da ordem de $9 \%{ }^{20}$. No Brasil, está em torno de II\%, ${ }^{14,2 \mid}$ no Estado do Rio de Janeiro em 
$9,1 \%{ }^{22}$ e em alguns municípios do interior de São Paulo em torno de 6,7\% $\%^{23}$. No entanto, nem todos os recém-nascidos de baixo peso necessitam de cuidados intensivos. Neste trabalho, encontrou-se uma média de $7 \%$ para a taxa de internações em UTI-Neonatal em relação ao número de nascimentos no Estado, 0 que resultou na projeção da necessidade de 885 leitos de UTI neonatal no estado (cerca de 3,8 para cada I. .000 nascimentos/ano). Swyer, no Canadá, calculou esta necessidade como de 4,7:1.000, sendo $15 \%$ de leitos intensivos $(0,7: 1.000)$ e $85 \%$ de semi-intensivos (4:1.000)24. Por outro lado, o Programa Perinatal de Wisconsin, EUA, calculou esta necessidade como de 2:1.000, ambos considerando como de $7 \%$ a taxa de nascimentos prematuros $^{25}$. Na Europa, Field relatou a necessidade de leitos intensivos como sendo de I, I:I.00026. Pelos dados aqui apresentados, no Estado do Rio de Janeiro, esta proporção parece estar próxima a 4:1000, o que corrobora a proposta da Sociedade Brasileira de Pediatria $(S B P)^{18}$, acima referida. Defendemos, entretanto, o critério de I/3 dos leitos como intensivos, ao invés dos $25 \%$ propostos pelaSBP, tendo em vista ser esta a composição predominante nas diversas unidades. Deste modo, para os 900 leitos neonatais necessários, calculamos como 300 intensivos e 600 semi-intensivos. Quanto à necessidade de leitos pediátricos, concluímos ser necessários algo em torno de 230 leitos em todo o Estado (75 intensivos e 155 semi-intensivos).

Além do número de leitos, a análise da eqüidade deve levar em consideração a distribuição geográfica e o percentual da população com acesso à medicina suplementar privada ou dependente exclusivamente da medicina pública (SUS). Dados fornecidos pela ABRAMGE ${ }^{13}$ dão conta de que no ano de 1996 a parcela da população com acesso à medicina suplementar privada era de cerca de 39.300 .000 beneficiários ( $42 \%$ medicina de grupo, $23 \%$ cooperativas médicas, $23 \%$ autogestão e I $2 \%$ seguradoras), para uma população de 157.070.163 habitantes, segundo o censo de 1996 (IBGE) "1 , o que corresponde a aproximadamente $25 \%$ da população. De posse destes dados e, embora considerando que este percentual possa ser um pouco maior nos grandes centros urbanos (de $30 \%$ a $35 \%$ na cidade do Rio de Janeiro) ${ }^{22}$ e menor no interior (em torno de 20\%), a aplicação da média de $25 \%$ nos levou a projeção de que seriam necessários em torno de 225 leitos privados e 675 públicos na área de UTINeonatal e 60 leitos privados e 170 do SUS em UTI-Pediátrica no âmbito do Estado do Rio de Janeiro.

Do ponto de vista global, o número de leitos de terapia intensiva oferecidos em todo Estado é da ordem de 1080 (60\% UTI, 40\% UI), o que corresponde a oferta de um leito para cada 3.227 crianças. Olhando-se sob o ponto de vista global, observa-se que estes números estariam próximos ao preconizado (I:3.000). No entanto, a primeira correção necessária diz respeito a distribuição dos leitos entre públicos e privados, ou seja, a capacidade de acesso da população a esta oferta. Considerados como de "acesso universal", os leitos oferecidos pelo SUS totalizam 616 (57\%) enquanto os do setor privado, considerados como de "acesso restrito" às famílias que dispõem de algum tipo de cobertura suplementar, totalizam $464(43 \%)$. Estes dados configuram um desequilíbrio entre oferta e demanda em ambos os setores, com menor proporção de leitos no SUS ( $57 \%$ da oferta para $75 \%$ da população) e maior na área privada ( $43 \%$ da oferta para $25 \%$ da população).

Realizando-se, no entanto, uma segunda leitura destes mesmos dados, desta vez considerando-se a distribuição geográfica dos leitos, observa-se uma concentração na mesorregião metropolitana do Rio de Janeiro, que compreende $93 \%$ dos leitos de todo o Estado, para $74 \%$ da população infantil (Tabela I). Estes números apontam uma clara desvantagem para o interior, que dispõe de apenas $7 \%$ dos leitos para $26 \%$ da população, destacando-se que as regiões da Baía da llha Grande, Centro-Sul e Noroeste Fluminense não dispõem de qualquer leito para uma população conjunta de 178.026 crianças de 0 a 14 anos de idade. Procedendo-se a esta mesma análise, agora sob o ponto de vista do número de leitos do SUS e do setor privado, observa-se que não há eqüidade na oferta, com clara desvantagem para a população dependente exclusivamente do SUS e uma situação mais confortável, mas também não ideal (exceto região do Médio Paraíba), mesmo para a parcela da população com acesso à medicina privada suplementar(Tabela 2).
Por outro lado, mesmo dentro da região metropolitana do Rio de Janeiro, não há eqüidade, pois a maioria está localizada na cidade do Rio de Janeiro, que dispõe de $73 \%$ dos leitos para $50 \%$ da população. Este mesmo quadro, quando analisado sob a ótica do número de leitos públicos e privados, repete as distorções, destacando-se que os municípios de Belfort Roxo, Guapimirim, Itaboraí, Itaguaí, Japeri, Magé, Mangaratiba Maricá, Paracambi, Queimados, São João de Meriti e Seropédica não dispõem de qualquer leito, público ou privado, para cerca de 500.000 crianças, com as demais regiões apresentando o mesmo padrão de carência de leitos do SUS e excesso no setor privado (Tabela 4), culminando com 0 observado no Município do Rio de Janeiro, que oferece um total de $4 \mathrm{I} 5$ leitos públicos e 319 privados, na proporção aproximada de ।:2.300 na área do SUS e 1:1.000 no setor privado. Portanto, na região metropolitana do Rio de Janeiro há grande variação, desde extrema carência nas regiões mais afastadas dos grande centros até uma oferta, em termos absolutos, bastante satisfatória na cidade do Rio de Janeiro.

Analisando-se em separado a cidade do Rio de Janeiro ( $73 \%$ dos leitos), observa-se a mesma distorção com a pior situação na AP 5.3. (Santa Cruz e Guaratiba), onde não há qualquer leito, público ou privado, para uma população de 104.883 crianças, situação pior do que em muitas regiões do interior do estado. A análise atenta da Tabela 5 permite constatar que os leitos existentes estão mal distribuídos em relação as zonas mais populosas da cidade, predominando no centro, zona norte ezona sul em detrimento das zonas suburbana e oeste. No entanto, este fato tem razões históricas compreensíveis, uma vez que os maiores hospitais públicos de referência encontram-se nas zonas mais antigas e centrais da cidade, enquanto os leitos privados desenvolveram-se pioneiramente nos hospitais terciários e com maiores recursos situados nas zonas sul e norte da cidade. Apesar da falta de eqüidade entre as áreas de planejamento de saúde, pode-se dizer que, de um modo global, não há no plano teórico carência de leitos de terapia intensiva para atender a população da cidade. Pode-se concluir, portanto, que a questão da qualidade da assistência na cidade do Rio de Janeiro parece deslocar-se da eqüidade na 
distribuição dos leitos para outros aspectos, como volume de atendimentos a pacientes de outras regiões mais carentes (cinturão metropolitano e interior do Estado); tipo de atendimento (neonatal ou pediátrico); acesso aos leitos já existentes através de um sistema de referência e transporte eficientes; e recursos de estrutura e processo das unidades em funcionamento. Embora perceba-se uma situação muito mais confortável no setor privado em relação ao número de leitos, com excesso de oferta em relação à demanda, há uma proporção satisfatória também na área do SUS, ficando as eventuais distorções do sistema por conta provavelmente dos fatores acima mencionados.

Pode-se ainda discutir a eqüidade na distribuição dos leitos entre as diversas regiões do Estado em relação ao tipo de atendimento, se neonatal ou pediátrico. Observa-se que a existência de unidades exclusivamente neonatais é uma característica quase que exclusiva da região metropolitana do Rio de Janeiro, que dispõe de 30 destas unidades (539 leitos) para um total de $66 \mathrm{UTIs}$ (45\%, vide Tabela I). 0 interior, por outro lado, possui apenas duas unidades exclusivamente neonatais (ambas na região do Médio Paraíba) para um total de oito UTIs (25\%). No interior do Estado, portanto, predominam as unidades mistas, que oferecem leitos neonatais (40 leitos) e pediátricos (2I leitos), provavelmente com a intenção de concentrar recursos e equacionar oferta e demanda (Tabela 3). Comportamento semeIhante, no entanto, observa-se também na região metropolitana em relação às unidades privadas, constatando-se que o número de leitos neonatais exclusivos é muito maior no setor público (343 x 196, vide Tabela 3), com predomínio de leitos mistos no setor privado $(93 \times 219)$, embora estes leitos mistos componham-se predominantemente de leitos neonatais. No SUS, dos 93 leitos mistos, 60 são neonatais (65\%), e no setor privado dos 219 existentes, 134 são neonatais (61\%). Nos EUA, Pollack ${ }^{27}$, estudando 289 unidades pediátricas, observou que 0 percentual de leitos neonatais é de $5 \%$ a $7 \%$, em forte contraste com o observado no estado do Rio de Janeiro.

Portanto, a soma de todos os leitos neonatais no Estado, considerando-se as unidades neonatais exclusivas e os leitos neonatais em unidades mistas, alcança 79 I ou $73 \%$ do total disponível e analisando-se os números da Tabela 3 conclui-se que, de um modo global, há excesso de oferta de leitos neonatais no setor privado, enquanto noSUS há pequeno déficit de leitos intensivos $(25 \%)$ e carência de cerca de $90 \%$ de leitos semiintensivos. Na verdade, não se pode considerar que o problema da assistência perinatal no Rio de Janeiro irá se resolver com a abertura de leitos neonatais intensivos, embora seja necessário reduzir o desequilíbrio entre a região metropolitana e o interior do Estado. Países com problemas semelhantes aos nossos, como a Índia, têm procurado investir em programas de assistência à gestante, treinamento de ressuscitação em sala de parto, unidades de nível II e unidades de nível III mais baratas. Naquele país, com 300 milhões de crianças ( $35 \%$ da população), taxa de mortalidade infantil de $73 \%$ e mortalidade neonatal de 47,2 \% existem I 50 UTIs de nível III e 450 de nível $\|^{28}$. O Estado do Rio de Janeiro está com uma taxa de mortalidade neonatal de $19,8 \%(1996, \text { SES })^{29}$ e conta com 32 unidades de nível Ill e mais $60 \%$ dos leitos das unidades mistas. Em nosso país, as autoridades de saúde também estão atentas à importância da atenção aos cuidados perinatais como estratégia para redução da mortalidade infantil e a SBP tem desenvolvido orientações claras em relação à melhoria do sistema ${ }^{30}$.

Considerando-se agora o número de leitos intensivos pediátricos, observa-se que em todo o Estado estão disponíveis 289 leitos ( $27 \%$ do total), sendo I 89 ligados ao SUS e 100 privados. Considerando-se os números anteriormente projetados, chega-se à conclusão de que seriam necessários em torno de 230 leitos intensivos pediátricos, aproximadamente 180 na área na área pública e 50 no setor privado. Conclui-se que, de um modo global, há excesso de oferta $(100 \%$ mais) de leitos intensivos pediátricos no setor privado e adequação no SUS. No entanto, novamente é necessário analisar estes dados sob o ponto de vista da distribuição regional. $O$ interior do Estado conta com 21 leitos pediátricos, sendo 10 do SUS e II privados para 901.446 crianças de 0 a I4 anos. Esta densidade populacional, no entanto, indicaria uma necessidade de 45 leitos na área pública e 15 leitos no setor privado, números não alcançados pelo SUS (déficit de $350 \%$ ) e parcialmente atendidos pela iniciativa privada (déficit de 35\%).

Na verdade, em alguns países há um sistema eficiente de assistência médica com um quantitativo número de leitos até menor do que o nosso, face a um serviço de referência e transporte mais organizado e provavelmentea diferenças na qualidade do processo. Enquanto no Estado do Rio de Janeiro a disponibilidade de leitos de UTI-Pediátrica (somente na área do SUS) é de cerca de I: 15.000 crianças, na Austrália e Nova Zelândia, com população semelhante ao do Estado (18.000.000 habitantes) há somente I0 UTIPs localizadas nas capitais, com uma relação de I:60.000, mas com uma política de assistência aparentemente eficiente, onde crianças com enfermidades de curta duração são admitidas nas unidades de adultos e crianças com problemas mais graves ou específicos são referidas para as UTls-Pediátricas existentes ${ }^{31}$. Em Ontário, Canadá, existem 0,8 leitos de UTI-Pediátrica para 100.000 habitantes (população geral) ${ }^{32}$, metade da oferta existente no Estado do Rio de Janeiro, se considerarmos apenas os leitos do SUS (I,9:100.000 habitantes).

Opercentual de leitos desativados $(n=65)$ em relação aos planejados $(n=1126)$ foi de $5,8 \%$. A análise de sua distribuição indica que a maioria (74\%) encontra-se no setor privado, que possui um total de 48 leitos desativados, contra apenas 17 no setor público. Tal fato se deve provavelmente ao excesso de oferta na área privada, que trabalha, de um modo geral, com grande ociosidade de leitos.

A maioria das unidades está localizada em hospitais gerais $(n=50,68 \%)$ ou pediátricos gerais (5,7\%), o que está de acordo com a tendência de localização destas unidades em hospitais terciários, que disponham de amplos recursos de diagnóstico e tratamento. Alocalização em clínicas de emergência pediátrica $(4,5 \%)$ está em declínio, pelos mesmos motivos. É natural observar que algumas unidades neonatais situem-se em maternidades e hospitais materno-infantis (I5,2\%), embora esta situação possa significar a ausência de determinados recursos de diagnóstico e tratamento, hoje essenciais, como certos recursos de diagnóstico por imagem, determinadas técnicas de laboratório ou assessoria de serviços médicos de outras especialidades, não disponíveis em 
maternidades e não acessíveis, principalmente no setor público.

Merece destaque ainda a constatação de que somente quatro unidades $(5,4 \%)$ encontram-se localizadas em hospitais universitários. Estas unidades oferecem em conjunto 46 leitos neonatais ( 19 intensivos, 27 semi-intensivos)e apenas quatro pediátricos. Tal situação, mais grave para $o$ atendimento pediátrico do que para o neonatal, coloca a universidade muito afastada de seu papel na formação de recursos humanos, o que pode ter um impacto significativo na qualidade da assistência. Esta realidade é diametralmente oposta ao que se observa em países mais avançados. Nos EUA, $76 \%$ das unidades pediátricas são ligadas a Centros Universitários e 81\% têm Residentes $\mathrm{R}^{6}$.

Em relação às propostas de melhoria na equiidade, a criação de uma central de vagas, medida simples e de implantação relativamente rápida, centralizaria as decisões sobre internações e aproveitamento de leitos, o que acoplado a um sistema de transporte eficiente, como é feito em muitas partes do mundo, poderia otimizar os recursos disponíveis. Outra solução seria a criação de novos leitos levando-se em consideração as carências regionais. Neste particular, vale ressaltar que na cidade do Rio de Janeiro há um excesso de cerca de 50 leitos neonatais intensivos em relação à demanda projetada pelo número de nascimentos por ano (o quantitativo de leitos semi-intensivos parece adequado), de modo que para atender a proposta de mais 250 leitos neonatais em todo o Estado propõe-se, além da criação de novos leitos, o remanejamento de leitos intensivos e recursos de hospitais das zonas centro e sul da cidade do Rio de Janeiro para regiões mais carentes e necessitadas, em hospitais já existentes, o que poderia reduzir significativamente os custos envolvidos, além de adequar melhor a demanda e oferta de leitos. Em relação à demanda por leitos pediátricos intensivos, que é menor, defendemos a criação de unidades mistas (com setores neonatais e pediátricos fisicamente distintos) em algumas cidades do interior e em certas regiões do cinturão metropolitano, sempre que $\circ$ quantitativo de leitos neonatais e pediátricos projetado for inferior ou iguala 10 , visando o aproveitamento máximo da estrutura a ser criada para $o$ atendimento a estes leitos. Quando a projeção indicar a necessidade de mais 10 leitos, seria adequada a criação de unidades neonatais e pediátricas em separado (Tabela 7).

\section{Conclusóes}

Com base nos resultados encontrados, pode-se concluir que: I) não há eqüidade na distribuição geográfica dos leitos intensivos no Estado, com concentração desproporcional na região metropolitana e grande carência no interior e desequilíbrio dentro da própria região metropolitana, com carência nas áreas de maior densidade populacional; 2) não há eqüidade no acesso da população aos leitos existentes no Estado como um todo, com déficit no setor público e adequação ou excesso no setor privado; 3 ) na cidade do Rio de Janeiro o número de leitos existentes, tanto públicos quanto privados, está em condições de atender às necessidades estimadas da população; 4) somente $5 \%$ das unidades encontram-se em hospitais universitários, o que representa uma redução na capacidade de formação e educação continuada dos recursos humanos, com potencial impacto negativo na qualidade da assistência; 5 ) melhoria na equiidade poderia ser obtida com a criação de uma central de vagas, de um sistema de transporte eficiente, de novos leitos e remanejamento de leitos para áreas mais carentes.

Os dados apresentados neste trabalho constituem parte da Tese de Doutoramento do autor principal: "Barbosa AP-Qualidade da Assistência em Tratamento Intensivo Neonatal e Pediátrico do Estado do Rio de Janeiro: Situação Atual e Propostas para melhoria", defendida pela UFRJ, em novembro de 1998.

\section{SUMMARY}

Neonatal and pediatric intensive CARE IN RIO dE JANEIRO: DISTRIBUTION OF BEDS AND ANALYSIS OF EQUITY

OBJECTIVES. To identify the pediatric ICUs at Rio de Janeiro, number of beds, geographical distribution, public or private nature, type of hospital and assistance, studying population demand and to propose measures for improving equity.

Methods: All ICUs of the State were visited from July 97 to June 98, identifying number of beds and average length of stay. With this information along with demographic data from IBGE, the necessity of beds were estimated, comparing availability and demand by region, and proposing improving equity strategies.

Results: 80 ICUs were identified (6 excluded), totaling 1080 beds; $60 \%$ intensive and $40 \%$ semi-intensive; $57 \%$ public and $43 \%$ private; $52 \%$ in exclusive neonatal ICUs; $14 \%$ in pediatric and $34 \%$ in mixed $(65 \%$ neonatal beds), totaling 79 I neonatal beds (73\%). The majority of ICUs (75\%) were part of general hospitals, $20 \%$ were in obstetric or obstetricpediatric hospitals, and only $5 \%$ were part of university centers; the majority were in metropolitan area (89\%), with $93 \%$ of beds for $74 \%$ of state children population, of whom, the majority were in Rio de Janeiro city (76\%), with $73 \%$ of beds for $37 \%$ of population, contrasting with the inner of the State, with only 8 units (II\%) and 79 beds (7\%) for $26 \%$ of children.

Conclusions: There is no equity in the distribution and accessibility to the available beds, with lack in public and excess in private sectors, a great concentration in the metropolitan area and only $5 \%$ of ICUs at university hospitals, recommending a policy of redistribution and allocation of new beds in more needy areas, associated with the creation of an admission center along with an efficient reference and transportation system. [Rev Assoc Med Bras 2002; 48(4): 303-II]

KEYWORDS: Intensive care. Public health. Equity. Health services accessibility. Statistics \& numerical data. Quality of health care.

\section{RefERÊnCIAS}

I. Thompson MH, Khot AS. Impact of neonatal intensive care. Arch Dis Child 1985;60:21 3-4.

2. Pollack MM, Yeh TS, Ruttimann UE, Holbrook PR; Fields Al. Evaluation of pediatric intensive care. Crit Care Med I 984; I 2:376-83.

3. Pollack MM, Alexander SR, Clarke N, Ruttimann UE; Tesselaar HM; Bachulis AC. Improved outcomes from tertiary center, pediatric intensive care: Astatewide comparison of tertiary and nontertiary care facilities. Crit Care Med 1991; 19:150-9.

4. Pollack MM, Katz RW, Ruttimann UE, Getson PR. Improving the outcome and efficiency of pediatric intensive care. Crit Care Med 1988; 16: I1-7.

5. Associação de Medicina Intensiva Brasileira. Projeto AMIB do Tamanho do Brasil: $I^{\circ}$ Censo Brasileiro de UTI's. São Paulo: AMIB; 1997. 
6. Barbosa AP, Cabral SA, Cardoso JL. Infraestrutura de atendimento nas UTIs públicas da cidade do Rio de Janeiro. In: II Encontro de Emergência e Tratamento Intensivo em Pediatria do Rio de Janeiro, 17 a 20 de outubro, Rio de Janeiro. Anais... Rio de Janeiro: SOPERJ. I990; p. I0. Sociedade de Pediatria do Estado do Rio de Janeiro.

7. Conselho Regional de Medicina, Grupo de Trabalho Materno-Infantil. Projeto de Assistência Materno-Infantil no Estado do Rio de Janeiro. CREMERJ, 1997.

8. Fundação Nacional de Saúde. Divisão Territorial com indicação das Regiões de Saúde [novembro de 1998]. Disponível no URL: http://www.funasa.gov.br

9. Fundação Instituto Brasileiro de Geografia e Estatística - IBGE. Divisão Territorial com indicação das Mesorregiões e Microrregiões Geográficas e Municípios [novembro de 1998]. Disponível no URL: http://www.ibge.gov.br

10. Secretaria Municipal de Urbanismo, Empresa Municipal de Informática e Planejamento IPLANRIO Coordenadoria de Informações da Cidade. Divisão Administrativa - Áreas de Planejamento, Regiões Administrativas e Bairros. Prefeitura da Cidade do Rio de Janeiro, 1997.

II. Fundação Instituto Brasileiro de Geografia e Estatística - IBGE. Censo Demográfico e Estimativas, 1996 [novembro de 1998]. Disponível no URL: http://www.ibge.gov.br

12. Ministério da Saúde. Planejamento e Dimensionamento. In: Equipamentos para estabelecimentos assistenciais de saúde. Brasília: Ministério da Saúde, 1994.

13. Associação Brasileira de Medicina de Grupo ABRAMGE. Dados e números da medicina de grupo Sistemas alternativos de saúde [no- vembro de 98]. Disponível no URL: http:// www.abrange.com.br

14. Departamento de Informática do SUS DATASUS. Sistema de Informações de Nascidos Vivos - SINASC [novembro de 1998]. Disponível noURL: http://www.datasus. gov.br

15. Ministério da Saúde. Portaria No 3.046/82. Estabelece parâmetros para planejamento assistencial a serem utilizados no INAMPS, 1982.

16. Departamento de Informática do SUS DATASUS. Sistema de Informações Hospitalares do SUS (SIH/SUS) [novembro de 1998]. Disponível no URL: http://www. datasus.gov.br

17. Ministério da Saúde. Portaria N³432/GM, de 12 de agosto de 1998. Estabelece critérios de classificação para as Unidades de Tratamento Intensivo - UTI. Diário Oficial da União $\mathrm{N}^{\circ}$ I54, Seção I, 13 de agosto, 1998.

18. Fiori RM. Regionalização da Assistência Perinatal. In: Miranda LEV and Lopes JMA, eds. Manual de Perinatologia. Rio de Janeiro: Sociedade Brasileira de Pediatria, 1991.

19. UNICEF - Fundo das Nações Unidas para a Infância. Situação Mundial da Infância 1994. Brasília: UNICEF; 1994.

20. Solés JA. Saúde reprodutiva e perinatal. In: Benguigui Y, Land S, Paganini J, and Yunes J, eds. Ações de Saúde Materno-Infantil a Nível Local. Washington: OPAS, 1997.

21. Nóbrega FJ. Antropometria, patologias e mal formações congênitas do recém-nascidobrasileiro e estudo de associação com algumas variáveis maternas.JPediatr (RioJ) I995; 59(2):3-I I4.

22. Silva RI, Theme Filha MM, Noronha CP. Sistema de informação sobre nascidos vivos na cidade do Rio de Janeiro 1993/1996. Informe Epidemiológico doSUS 1997;Ano VI(2):33-48.
23. Costa CE. O peso ao nascer e a declaração de nascido vivo: um estudo epidemiológico. Universidade de São Paulo, 1996. Dissertação de Mestrado. São Paulo.

24. Swyer PR. Organisation of perinatal/neonatal care. Acta Paediatr Suppl 1993;385: I - 8.

25. Fanaroff AA, Graven SN. Perinatal services and resources. In: Fanaroff $\mathrm{AA}$ and Martin RJ. Neonatal-Perinatal Medicine. 5 ed. St. Louis: Mosby Year Book, 1992.

26. Field DS. The demand for neonatal intensive care. BMJ I 989;299(67 I I): I 305-8.

27. Pollack MM, Cuerdon TC, Getson PR. Pediatric intensive care units: Results of a national survey. Crit Care Med 1993;2 I (4): 607-I4.

28. Vidyasagar D, Singh M, Bhakoo, ON, et al. Evolution of neonatal and pediatric critical care in India. Crit Care Clinics 1997; I 3(2):33 I -46.

29. Secretaria de Estado de Saúde. Taxa de mortalidade neonatal no Estado do Rio de Janeiro [novembro de 1998]. Disponível no URL: http://www.saude.rj.gov.br

30. Fiori RM. Regionalização da assistência perinatal. In: Segre CAM and et al. Manual de Neonatologia. Rio de Janeiro: Sociedade Brasileira de Pediatria, 1997.

31. Dobb GD. Intensive Care in Australia and New Zealand: No Nonsense “Down Under”. Crit Care Clinics 1998; I 3(2):299-3 I6.

32. Sibbald WJ, Singh T. Critical care in Canada: The north american difference. Crit Care Clinics 1997; I 3(2):347-62.

Artigo recebido: $28 / 11 / 2001$ Aceito para publicação: 16/05/2002 